\title{
UNE LETTRE DE PAUL LE COINTE SUR L'AMAZONIE
}

Nos lecteurs connaissent Paul Le Cointe par ses beaux travaux sur l'Amazonie, ils le connaitront mieux encore par l'admirable lettre qu'il nous envoie et dont nous avons l'indiscrétion de publier les passages essentiels. Mais qui ne nous en remerciera? - F. B.

Ẽvidemment, par ancienneté, je ne peux pas faire autrement que de connaître un peu l'Amazonie et, faute de concurrents, on m'attribue généreusement le privilège de la connaître dans tous ses détails, sous tous ses aspects. Je sais trop bien qu'il n'y a pas lieu de prendre au sérieux ces exagérations. La réalité est la suivante.

En ı 891 , j'étais à l'Institut Chimique de Nancy, préparateur au laboratoire du directeur, le grand savant Albin Haller. Sur ma demande et la proposition du "Comité des travaux historiques et scientifiques ", j'obtins d'être chargé de mission gratuite en Amazonie brésilienne (Arrêté du ministre Léon Bourgeois, 23 nov. $189 \mathrm{r}$ ).

Je devais avoir trois compagnons, mes amis, dont E.-F. Gautier, le futur Saharien ; pour divers motifs (en réalité, je pense, parce que j'étajs le plus jeune et que mon plan leur paraissait irréalisable), ils me lâchèrent avant le départ de Paris fixé au 28 novembre; l'un d'eux, seul, m'accompagna jusqu'au quai Sully où je m'embarquai sur un petit canot à vapeur dont l'achat avait absorbé le plus clair de mes ressources et que, à titre d'entraînement, sans doute, je conduisais sans aucune aide, jusqu'au Havre, pour prendre le paquebot anglais qui devait nous transporter jusqu'a l'Amazone. Les premiers temps furent un peu durs; il me fallut absorber et digérer, autant que possible, les rôles qui avaient été réservẻs aux divers membres de la " mission », suivant les aptitudes de chacun de nous, et voilà cinquante-six ans rue, l'un après l'autre, je biffe, notant " exécuté ", les paragraphes de mon fameux plan, sans espoir, déjà, d'arriver au dernier.

Je me suis intéressé à tout ce qui constitue l'Amazonie : nature et population, mais qu'il s'agisse de simples voyages, ou d'exploration, des travaux les plus divers, géographiques ou agricoles, d'expériences de laboratoire ou de publications, de collections touchant la faune, la flore ou les minéraux, j'ai toujours eu en vue d'étudier le résultat économique pouvant découler de mes observations soigneusement notées et classées.

Quand, après avoir vécu trente années dans différentes régions du bassin du Rio Mar, entre l'altitude de o mètre et 5 ooo mètres, j'ai écrit mon ouvrage L'Amazonie brésilienne (2 vol. ill. in $\left.-8^{\circ}\right)$, édité à Paris en 
rgax, par la "Sociétẻ d'Éditions géographiques, maritimes et coloniales ", $j$ 'habitais Obidos, très petite ville située sur les rives du fleuve, à I $000 \mathrm{ki}$ lomètres en amont de son embouchure, à peu de distance d'une plantation de cacao et de caoutchouc, et d'une petite ferme d'élevage qui m'appartenaient; j'étais donc placé comme en face du sujet à traiter, à ane distance suffisante du centre de toutes ses activités, Bélem, la capitale de l'Etat du Para, isolé ainsi de toute influence directe d'opinions improvisées et contradictoires, pouvant conserver rigoureusement l'indispensable indépendance de jugement d'un observateur étranger, percevant nettement, avec les détails, l'ensemble formé par leur réunion.

Après avoir publié quelques cartes géographiques, j'ai pu me risquer a tenter une description générale, non seulement physique, mais aussi économique et sociale, usant de la plus grande franchise, sans ménager de nombreuses critiques largement documentées, ni les suggestions corrélatives que l'étude d'un pays encore dans son enfance, malgré plusieurs siècles de colonisation, ne pouvait manquer de provoquer.

J'ai eu la très grande satisfaction de voir mon Amazonie brésilienne reçuc partout, et surtout au Brésil, comme je n'aurais jamais osé l'espérer. J'ai publié ensuite, mais en portugais, d'autres études, entrant dans le détail de quelques points les plus intéressants de mon plan primitif : L'État du Para, la terre, l'eau et l'air ; Dictionnaire des Plantes Utiles ; La Culture du Cacao ; Le Caoutchouc en Amazonie ; Les Graines Oléagineuses, Résines et Essences ; Bulletin de l'École de Chimie Industrielle (créée et dirigée pendant dix ans); Les crues croissantes de l'Amazone et leur menace, etc...

Malheureuscment, au lieu du brillant développement que je croyais d'abord être le très proche avenir de l'Amazonie en raison de ses magnifiques possibilités, je ne peux pas m'empêcher de constater un véritable recul, a tous points de vue, et, ce qui est grave, surtout au moral, et je ne vois pas, eu égard à la situation mondiale, de quelle manière on pourrait l'enrayer. L'Amazonie, qui n'a pas été touchée par la guerre dans ses organes essentiels, et vers laquelle il y a eu plutòt un afflux d'argent dont clle n'a su tirer aucun parti, doit aujourd'hui faire face à une terrible crise économique tout en n'ayant guère pour guides que des mencurs politiques incompétents, trop souvent même simples arrivistes sans scrupules; je la vois se débattre si maladroitement que je ne la crois pas capable de s'en sortir par ses propres moyens, j'ai plutôt foi dans le fait que les pays qui, comme le nôtre, ont tant souffert auront l'énergie nécessaire pour reprendre pied, et ce sera leur retour à la normalité qqui obligera l'Amazonie à poursuivre enfin sa marche vers le progrès qui l'attend depuis si longtemps.

Bélem, 5 avril 19 !r.

Paul Le Cornte.

Le Gérant : G. Mignot. 